\title{
Integrability of a nonlinear evolution equation derived from isoperimetric plane curve motion
}

\author{
J. C. Brunelli* \\ Departamento de Física, CFM \\ Universidade Federal de Santa Catarina \\ Campus Universitário, Trindade, C.P. 476 \\ CEP 88040-900 \\ Florianópolis, SC, Brazil
}

\begin{abstract}
We provide a geometrical interpretation for the series of transformations used by Sakovich to map the third-order nonlinear evolution equation obtained by Chou and Qu to the mKdV equation. We also discuss its bi-Hamiltonian integrability as well as integrable equations associated with this system.
\end{abstract}

PACS: 02.30.Ik; 02.30.Jr; 05.45.-a

Keywords: Integrable models; Bi-Hamiltonian systems;

*jcbrunelli@gmail.com 


\section{Introduction:}

Starting with the work of Hasimoto [1] the study of the connection between integrable models and the dynamics of space curves has drawn a lot of interest. Motion of these curves and the associated integrable models were considered in Euclidean space $E^{3}$ [2, 3, 4, $S^{2}$ and $S^{3}$ spaces [5] and Minkowski space [6, 7]. However, integrable equations associated with the motion of plane curves are more scarce [8, 9]. This has motivated Chou and Qu [10, 11] to study motions of plane curves in other background geometries, replacing the Euclidean geometries by Klein ones. As a result they have shown that KdV, Harry-Dym and Sawada-Kotera hierachies, among other nonlinear equations, arise in an natural way from the motions of plane curves in affine, centroaffine and similarity geometries. In this process they also found a new equation associated with the $\mathrm{mKdV}$ equation, namely (see [10], last equation on page 31 ),

$$
u_{t}=\frac{1}{2}\left(\left(u_{x x}+u\right)^{-2}\right)_{x} .
$$

Chou and $\mathrm{Qu}$ did not investigated if this equation (from now on the CQ equation) arises from an AKNS- or the WKI-scheme of inverse scattering transformation, its integrability was investigated by Sakovich [12]. Through a chain of Miura-type transformations he related equation (1) with the mKdV equation. Firstly, (1) can be transformed to

$$
v_{t}=\frac{1}{2} v^{2}\left(\left(v^{2}\right)_{x x}+v^{2}\right)_{x}
$$

by the transformation

$$
(x, t, u(x, t)) \mapsto(x, t, v(x, t)): v=-\left(u_{x x}+u\right)^{-1}
$$

Then we perform two successive transformations

$$
\begin{aligned}
(y, t, w(y, t)) & \mapsto(x, t, v(x, t)): x=w, v=w_{y}, \\
(y, t, w(y, t)) & \mapsto(y, t, z(y, t)): z=w_{y},
\end{aligned}
$$

to obtain the $\mathrm{mKdV}$ equation

$$
z_{t}=z_{y y y}+\frac{3}{2} z^{2} z_{y}
$$

where $z(y, t)=v(x, t)$. Also, in [12] a zero curvature representation with an essential parameter was obtained as well as the following second-order recursion operator

$$
\bar{R}=\frac{1}{u_{x x}+u} \partial \frac{1}{u_{x x}+u}\left(\partial+\partial^{-1}\right) .
$$

It is well known that integrable equations also possess a bi-Hamiltonian structure which yields 
a recursion operator in the form $\mathcal{D}_{2} \mathcal{D}_{1}^{-1}$. So, going backwards Ren and Alatancang [13] proved the hereditary property of (6) and by a decomposition of it obtained the bi-Hamiltonian structure as well as a hierarchy of equations, associated with (1).

In this paper we point out, in Section 2, that the series of transformations (3) and (4) mapping the $\mathrm{CQ}$ equation (11) to the $\mathrm{mKdV}$ equation (5) has a nice geometrical interpretation which can be used to justify connections between other equations. In Section 3 the bi-Hamiltonian structure, and consequently the recursion operator $R$, is derived directly from a Lagrangian representation of (1). In fact, the original derivation of Ren and Alatancang for this bi-Hamiltonian structure relies on the knowledge of the bi-Hamiltonian structure of a system related to (1) by a Miura map (obtained previously by Olver and Rosenau [14]) and of the recursion operator (6). Therefore, their derivation is more a map between structures than a derivation from first principles. For completeness, in Section 4, we obtain the hierarchy of equations, some symmetries and equations related to (1). We discuss the relation among these equations. The conclusions are presented in Section 5 .

\section{Plane Curve Motion:}

Let be a closed smooth curve in the plane, parametrized by an arbitrary parameter $\alpha$, where $\alpha \in[0,1]$, and let $\mathbf{r}(\alpha, t)$ represent the position of a point on the curve at the time $t$. Along the curve the arc-length is $s(\alpha, t)=\int_{0}^{\alpha} \sqrt{g\left(\alpha^{\prime}, t\right)} d \alpha^{\prime}$ and we can use $s$ as a parameter as well (this is called Lagrangian description of the curve). The metric on the curve is $g=\mathbf{r}_{\alpha} \cdot \mathbf{r}_{\alpha}$ and we have that $d / d \alpha=\sqrt{g} d / d s$. At a point on the curve the unit tangent and normal vectors, defined by $\mathbf{t}(s, t)=\mathbf{r}_{s}$ and $\mathbf{n}(s, t)=\frac{1}{\kappa} \mathbf{r}_{s s}$, respectively, satisfy the Serret-Frenet equations

$$
\left(\begin{array}{l}
\mathbf{t} \\
\mathbf{n}
\end{array}\right)_{s}=\left(\begin{array}{cc}
0 & \kappa \\
-\kappa & 0
\end{array}\right)\left(\begin{array}{l}
\mathbf{t} \\
\mathbf{n}
\end{array}\right)=i \sigma_{2} \kappa\left(\begin{array}{l}
\mathbf{t} \\
\mathbf{n}
\end{array}\right),
$$

where $\kappa(s, t)$ is the curvature. The dynamics of the points of the curve are specified by

$$
\mathbf{r}_{t}=F \mathbf{n}+G \mathbf{t}
$$

where the normal and tangential velocities $F$ and $G$ are functions of the curvature $\kappa$. Now we make the further assumption that the perimeter $L=\oint d s$ of the closed curve remains constant in time, i.e., we assume an isoperimetric plane curve motion. Therefore, from the metric evolution

$$
g_{t}=2 \mathbf{r}_{\alpha} \cdot \mathbf{r}_{t \alpha}=2 g\left(G_{s}-\kappa F\right),
$$

we get

$$
\frac{d L}{d t}=\int_{0}^{1} \sqrt{g\left(\alpha^{\prime}, t\right)} d \alpha^{\prime}=\oint\left(G_{s}-\kappa F\right) d s
$$


and $d L / d t=0$ if $G_{s}=\kappa F$. This implies that the arc-length and time derivatives commute, $\left[\partial_{s}, \partial_{t}\right]=0$. The time evolution of the tangent and normal vectors gives

$$
\left(\begin{array}{c}
\mathbf{t} \\
\mathbf{n}
\end{array}\right)_{t}=\left(\begin{array}{cc}
0 & F_{s}+\kappa G \\
-F_{s}-\kappa G & 0
\end{array}\right)\left(\begin{array}{l}
\mathbf{t} \\
\mathbf{n}
\end{array}\right)=i \sigma_{2}\left(F_{s}+\kappa G\right)\left(\begin{array}{l}
\mathbf{t} \\
\mathbf{n}
\end{array}\right),
$$

and the compatibility condition between (7) and (9),

$$
\left(\begin{array}{l}
\mathbf{t} \\
\mathbf{n}
\end{array}\right)_{t s}=\left(\begin{array}{l}
\mathbf{t} \\
\mathbf{n}
\end{array}\right)_{s t}
$$

yields the following nonlinear evolution equation for the curvature

$$
\kappa_{t}=F_{s s}+\kappa_{s} \partial_{s}^{-1}(\kappa F)+\kappa^{2} F
$$

which can also be written as $\kappa_{t}=R F$ with

$$
R=\partial_{s}^{2}+\kappa_{s} \partial_{s}^{-1} \kappa+\kappa^{2} .
$$

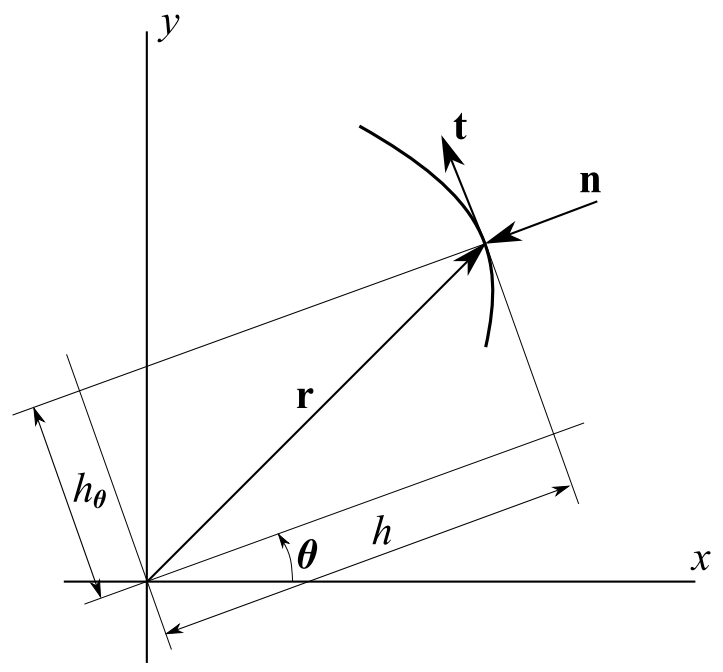

Figure 1

If we choose $F=-\kappa_{s}$ in (10) we obtain the $\mathrm{mKdV}$ equation,

$$
\kappa_{t}=-\kappa_{s s s}-\frac{3}{2} \kappa^{2} \kappa_{s}
$$

and we recognize (11) as the $\mathrm{mKdV}$ recursion operator. However, at this point let us change the curve description and parametrization. For a curve with positive curvature (uniformly convex) we introduce the support function $h(\theta, t)$, defined as

$$
h(\theta, t)=-\mathbf{r} \cdot \mathbf{n}
$$


where $\theta$, called the normal angle of the curve, is the angle between the unit normal $\mathbf{n}$ and the positive $x$-axis [15]. In the following let $\mathbf{r}=\mathbf{r}(\theta, t)$ and from the Figure 1 we have $\mathbf{n}=-(\cos \theta, \sin \theta)$ and $\mathbf{t}=(-\sin \theta, \cos \theta)$. For the support function $h(\theta, t)$ we obtain

$$
\begin{aligned}
h & =x \cos \theta+y \sin \theta \\
h_{\theta} & =-x \sin \theta+y \cos \theta,
\end{aligned}
$$

and $\mathbf{r}=(x, y)$ can be obtained form $h$ using

$$
\begin{aligned}
& x=h \cos \theta-h_{\theta} \sin \theta \\
& y=h \sin \theta+h_{\theta} \cos \theta .
\end{aligned}
$$

From this last equation we obtain

$$
\left|\mathbf{r}_{\theta}\right|=h_{\theta \theta}+h
$$

and since $d / d s=\left|\mathbf{r}_{\theta}\right|^{-1} d / d \theta$ the Serrat-Frenet formula

$$
\frac{d \mathbf{t}}{d s}=\left|\mathbf{r}_{\theta}\right|^{-1} \frac{d \mathbf{t}}{d \theta}=\kappa \mathbf{n}
$$

yields a neat expression for the curvature in terms of the support function

$$
\kappa=\frac{1}{h_{\theta \theta}+h} .
$$

Finally, from (8) the normal speed of $\mathbf{r}$ is given by $\mathbf{n} \cdot \mathbf{r}_{t}=-\kappa_{s}$ and therefore

$$
\begin{aligned}
h_{t} & =\kappa_{s}=\left|\mathbf{r}_{\theta}\right|^{-1} \kappa_{\theta}=\frac{1}{2}\left(\kappa^{2}\right)_{\theta} \\
& =\frac{1}{2}\left(\left(h_{\theta \theta}+h\right)^{-2}\right)_{\theta},
\end{aligned}
$$

which is the equation (1). An evolution equation for the curvature, in terms of the normal angle $\theta$, instead of the arc-length as in (12), can be obtained. From (14)

$$
\kappa_{t}=-\kappa^{2}\left(h_{\theta \theta t}+h_{t}\right),
$$

and after using (15)

$$
\kappa_{t}=-\frac{1}{2} \kappa^{2}\left(\left(\kappa^{2}\right)_{\theta \theta}+\kappa^{2}\right)_{\theta}
$$

It is well know that a curve is determined by its curvature up to a rigid motion [15], therefore, there is a formal equivalence between (16) and (15) (and between (8) and (12)) and the connection is made by (14). In other words the equation (15) and the curvature integrable equation (16) are equivalent. Now we are in position to justify geometrically the transformations (3) and (4) if we 
make the identifications $h(\theta, t) \leftrightarrow u(x, t), \kappa(s, t) \leftrightarrow z(y, t), \kappa(\theta, t) \leftrightarrow v(x, t), \theta \leftrightarrow x$ and $s \leftrightarrow y$ and recognize the equivalence between the equations (15) $\leftrightarrow$ (11), (12) $\leftrightarrow$ (5) and (16) $\leftrightarrow$ (22). The transformation (3) is simply (14) and the transformation (44) is the inverse transformation from (12) to (16) (projection (13) and change of parametrization from $s$ to $\theta$ ). The recursion operator (6) defines the curvature evolution under arc-length conserving dynamics and can be obtained from (11) using the identifications given above.

\section{Bi-Hamiltonian Structure:}

Let us study the integrability of (15) from a Hamiltonian point of view. Since the curvature plays a prominent role let us write (15) as

$$
h_{t}=\frac{1}{2}\left(\kappa^{2}\right)_{\theta}
$$

and where $\kappa$ satisfies (16) which can also be rewritten as

$$
\left(\kappa^{-1}\right)_{t}=\frac{1}{2}\left(\left(\kappa^{2}\right)_{\theta \theta}+\kappa^{2}\right)_{\theta} .
$$

Let us observe that the basic field is $h$ and that $\kappa$ is a placeholder used to make expressions more compact (see [16]). The equations (16) and (18) are not being viewed as nonlinear evolution equations but just as expressions for the time derivative of the placeholder $\kappa$, however, they can be interpreted as the Casimir equation for the so called modified compacton hierarchy (see equations $(27)$ and (28) in [14]). Also, we will consider $\theta$-periodic solutions, therefore, $\theta \in[0,2 \pi]$ and $h(\theta, t)=$ $h(\theta+2 \pi, t)$. We denote by $\partial \equiv \partial_{\theta}$ the differential operator with respect to $\theta$ with skew-adjoint inverse $\partial^{-1} \equiv \partial_{\theta}^{-1}$. Whenever the nonlocal operator $\partial^{-1}$ is used we have $\partial \partial^{-1}=\partial^{-1} \partial=I$, $\partial^{\dagger}=-\partial$ and $\left(\partial^{-1}\right)^{\dagger}=-\partial^{-1}$. We define the anti-derivative $\partial^{-1}$ as $\int_{0}^{\theta} d \theta^{\prime}$.

Equation (17) can be obtained from a variational principle, $\delta \int d t d \theta \mathcal{L}$, from the Lagrangian density

$$
\mathcal{L}=\kappa-h_{t} \partial^{-1} \kappa^{-1}
$$

This is a first order Lagrangian density and we can use the Dirac's theory of constraints [17] to obtain the Hamiltonian and the Hamiltonian operator associated with (19). The Lagrangian is degenerate and the primary constraint is obtained to be

$$
\Phi=\pi+h_{\theta}+\left(\partial^{-1} h\right)
$$

where $\pi=\partial \mathcal{L} / \partial h_{t}$ is the canonical momentum. The total Hamiltonian is

$$
H_{T}=\int d \theta\left(\pi h_{t}-\mathcal{L}+\lambda \Phi\right)=\int d \theta\left[-\kappa+\lambda\left(\pi+h_{\theta}+\left(\partial^{-1} h\right)\right)\right],
$$


where $\lambda$ is a Lagrange multiplier field. From the canonical Poisson bracket relation

$$
\left\{h(\theta), \pi\left(\theta^{\prime}\right)\right\}=\delta\left(\theta-\theta^{\prime}\right)
$$

with all others vanishing, we require the primary constraint to be stationary under time evolution,

$$
\left\{\Phi(\theta), H_{T}\right\}=0
$$

to determine the Lagrange multiplier field $\lambda$ in (21) and to find out that the system has no further constraints.

The canonical Poisson bracket relation (22) yields

$$
K\left(\theta, \theta^{\prime}\right) \equiv\left\{\Phi(\theta), \Phi\left(\theta^{\prime}\right)\right\}=\left(\partial_{\theta}+\partial_{\theta}^{-1}\right) \delta\left(\theta-\theta^{\prime}\right)-\left(\partial_{\theta^{\prime}}+\partial_{\theta^{\prime}}{ }^{-1}\right) \delta\left(\theta^{\prime}-\theta\right)
$$

and we find that the constraint (20) is second class. The Dirac bracket between the basic variables is

$$
\left\{h(\theta), h\left(\theta^{\prime}\right)\right\}_{D}=\left\{h(\theta), h\left(\theta^{\prime}\right)\right\}-\int d \theta_{1} d \theta_{2}\left\{h(\theta), \Phi\left(\theta_{1}\right)\right\} J\left(\theta_{1}, \theta_{2}\right)\left\{\Phi\left(\theta_{2}\right), h\left(\theta^{\prime}\right)\right\}=J\left(\theta, \theta^{\prime}\right),
$$

where $J$ is the inverse of the Poisson bracket of the constraint (23),

$$
\int d \theta^{\prime \prime} K\left(\theta, \theta^{\prime \prime}\right) J\left(\theta^{\prime \prime}, \theta^{\prime}\right)=\delta\left(\theta-\theta^{\prime}\right)
$$

From this last equation we get

$$
2\left(\partial+\partial^{-1}\right) J\left(\theta, \theta^{\prime}\right)=\delta\left(\theta-\theta^{\prime}\right)
$$

or

$$
J\left(\theta, \theta^{\prime}\right)=\mathcal{D}_{1} \delta\left(\theta-\theta^{\prime}\right)
$$

where

$$
\mathcal{D}_{1}=\frac{1}{2}\left(\partial+\partial^{-1}\right)^{-1}
$$

We now set the constraint (20) strongly to zero in (21) to obtain

$$
H_{2}=-\int d \theta \kappa
$$

and the equation (17) can be written in the Hamiltonian form as

$$
h_{t}=\mathcal{D}_{1} \frac{\delta H_{2}}{\delta h}
$$


From (18) it is straightforward to show that the charge

$$
H_{1}=\int d \theta \frac{1}{\kappa}
$$

is also conserved. Therefore, the equation (17) can be written in the Hamiltonian form as

$$
h_{t}=\mathcal{D}_{2} \frac{\delta H_{1}}{\delta h}
$$

where we have defined

$$
\mathcal{D}_{2}=\kappa \partial \kappa
$$

This Hamiltoninan structure is manifestly skew symmetric. Using the expansion

$$
\left(\partial+\partial^{-1}\right)^{-1}=\sum_{n=0}^{\infty}(-1)^{n} \partial^{-(2 n+1)}
$$

it is easy to show that $\mathcal{D}_{1}$ is also skew symmetric. Jacobi identity for these structures as well as their compatibility follows from standard method of prolongation [18. We can construct the two bivectors associated with the two structures as

$$
\begin{aligned}
& \Phi_{\mathcal{D}_{1}}=\frac{1}{2} \int d \theta\left\{\phi \wedge \mathcal{D}_{1} \phi\right\}=\frac{1}{4} \int d \theta \phi \wedge\left[\left(\partial+\partial^{-1}\right)^{-1} \phi\right], \\
& \Phi_{\mathcal{D}_{2}}=\frac{1}{2} \int d \theta\left\{\phi \wedge \mathcal{D}_{2} \phi\right\}=\frac{1}{2} \int d \theta \kappa^{2} \phi \wedge \phi_{\theta} .
\end{aligned}
$$

where $\phi$ is the univector corresponding to the one-form $d h$. Using the prolongation relations,

$$
\begin{aligned}
& \operatorname{pr} \vec{v}_{\mathcal{D}_{1} \phi}\left(\kappa^{2}\right)=-\kappa^{3} \phi_{\theta} \\
& \operatorname{pr} \vec{v}_{\mathcal{D}_{2} \phi}\left(\kappa^{2}\right)=-2 \kappa^{3}\left[\left(\partial+\partial^{-1}\right) \kappa(\kappa \phi)_{\theta}\right]_{\theta}
\end{aligned}
$$

we show that the prolongation of the bivector $\Phi_{\mathcal{D}_{2}}$ vanishes,

$$
\operatorname{pr} \vec{v}_{\mathcal{D}_{2} \phi}\left(\Phi_{\mathcal{D}_{2}}\right)=0
$$

implying that $\mathcal{D}_{2}$ satisfies Jacobi identity. Using (29), it also follows that

$$
\operatorname{pr} \vec{v}_{\mathcal{D}_{1} \phi}\left(\Phi_{\mathcal{D}_{2}}\right)+\operatorname{pr} \vec{v}_{\mathcal{D}_{2} \phi}\left(\Phi_{\mathcal{D}_{1}}\right)=0
$$

showing that $\mathcal{D}_{1}$ and $\mathcal{D}_{2}$ are compatible. Namely, not only are $\mathcal{D}_{1}, \mathcal{D}_{2}$ genuine Hamiltonian structures, any arbitrary linear combination of them is as well. As a result, the dynamical equation (17) is bi-Hamiltonian and, consequently, is integrable [18, 19]. Also, the bi-Hamiltonian structures (24) 
and (27) provide us with a natural recursion operator defined by

$$
R=\mathcal{D}_{2} \mathcal{D}_{1}^{-1}
$$

or

$$
R=2 \kappa \partial \kappa\left(\partial+\partial^{-1}\right)
$$

which is exactly the recursion operator $\bar{R}$, given by ([6), obtained by Sakovich [12].

\section{Hierarchy of Equations:}

From the bi-Hamiltonian structure obtained in the previous section we can naturally define a hierarchy of commuting flows by

$$
h_{t_{n}}=K_{n}[h]=\mathcal{D}_{1} \frac{\delta H_{n+1}}{\delta h}=\mathcal{D}_{2} \frac{\delta H_{n}}{\delta h}, \quad n=1,2,3, \ldots
$$

For $n=1$ we obtain (17). From (30) we have

$$
\frac{\delta H_{n+1}}{\delta h}=R^{\dagger} \frac{\delta H_{n}}{\delta h}
$$

where

$$
R^{\dagger}=\left(\mathcal{D}_{2} \mathcal{D}_{1}^{-1}\right)^{\dagger}=2\left(\partial^{-1}+\partial\right) \kappa \partial \kappa
$$

is the adjoint of $R$. Using (31) recursively we obtain an infinite set of conserved Hamiltonians

$$
\begin{aligned}
H_{1} & =\int d \theta \frac{1}{\kappa} \\
H_{2} & =-\int d \theta \kappa \\
H_{3} & =-\frac{1}{2} \int d \theta\left(\kappa^{3}-4 \kappa \kappa_{\theta}^{2}\right) \\
H_{4} & =-24 \int d \theta\left(3 \kappa^{5}+20 \kappa^{3} \kappa_{\theta \theta}^{2}+15 \kappa^{4} \kappa_{\theta \theta}+\kappa^{4} \kappa_{\theta \theta \theta \theta}\right) \\
H_{5} & =-2 \int d \theta\left[\frac{5}{16} \kappa^{7}+\frac{35}{12} \kappa^{6} \kappa_{\theta \theta}+7\left(\frac{13}{120} \kappa^{6} \kappa_{\theta \theta \theta \theta}+\frac{27}{20} \kappa^{5} \kappa_{\theta \theta}^{2}\right)\right. \\
& \left.-\frac{28}{15} \kappa^{5} \kappa_{\theta \theta \theta}^{2}-\frac{21}{2} \kappa^{4} \kappa_{\theta}^{2} \kappa_{\theta \theta \theta \theta}+\frac{21}{2} \kappa^{4} \kappa_{\theta \theta}^{3}+\frac{16}{45} \kappa^{6} \kappa_{\theta \theta \theta \theta \theta \theta}\right]
\end{aligned}
$$


These charges can also be obtained using the fact that

$$
H_{n}=\operatorname{Tr} R^{\frac{2 n-3}{2}}, \quad n=1,2,3, \ldots,
$$

where "Tr" is the Adler's trace [20]. We also obtained (32) in this way using our program PSEUDO [21].

The corresponding first flows associated with (32) are

$$
\begin{aligned}
h_{t_{1}} & =\frac{1}{2}\left(\kappa^{2}\right)_{\theta} \\
h_{t_{2}} & =\left(\frac{3}{4} \kappa^{2}+2 \kappa^{3} \kappa_{\theta \theta}+\kappa^{2} \kappa_{\theta}^{2}\right)_{\theta} \\
h_{t_{3}} & =\left(\frac{5}{4} \kappa^{6}+15 \kappa^{4} \kappa_{\theta}^{2}+10 \kappa^{5} \kappa_{\theta \theta}+18 \kappa^{4} \kappa_{\theta \theta}^{2}+24 \kappa^{4} \kappa_{\theta} \kappa_{\theta \theta \theta}+4 \kappa^{5} \kappa_{\theta \theta \theta \theta}+2 \kappa^{2} \kappa_{\theta}^{4}+32 \kappa^{3} \kappa_{\theta}^{2} \kappa_{\theta \theta}\right)_{\theta} \\
& \vdots
\end{aligned}
$$

Since every symmetry of an integrable model defines another integrable model we can go further in the study of the hierarchy of equations of our system through a symmetry study of Eq. (17). Using the Lie's algorithm (assisted by the computer algebra system program GeM [22]) we have obtained the following point and first higher order symmetry generators in evolutionary form

$$
\begin{aligned}
& X_{1}=h_{\theta} \frac{\partial}{\partial h}, \\
& X_{2}=\sin \theta \frac{\partial}{\partial h}, \\
& X_{3}=\cos \theta \frac{\partial}{\partial h}, \\
& X_{4}=-\frac{1}{2}\left(\kappa^{2}\right)_{\theta} \frac{\partial}{\partial h}, \\
& X_{5}=\left[-\frac{t}{2}\left(\kappa^{2}\right)_{\theta}+\frac{h}{3}\right] \frac{\partial}{\partial h} .
\end{aligned}
$$

Of course the flow associated with $X_{4}$ is in the hierarchy (33). The flow associated with $X_{2}$ can be obtained if we allow in (30) the value $n=0$. In fact, from

$$
h_{t_{0}}=\mathcal{D}_{1} \frac{\delta H_{1}}{\delta h},
$$


where $\delta H_{1} / \delta h=1$, and after using (28) and $\left(\partial^{-1} 1\right)=\theta^{n} / n$ ! we obtain

$$
h_{t_{0}}=\frac{1}{2}\left(\partial+\partial^{-1}\right)^{-1} \cdot 1=\frac{1}{2} \sum_{n=0}^{\infty}(-1)^{n}\left(\partial^{-(2 n+1)} 1\right)=\frac{1}{2} \sin \theta .
$$

From the flow $X_{1}$ we take $h_{t}=h_{\theta}$ as a seed equation and the recursion procedure $h_{t}=\left(R^{-1}\right)^{n} h_{\theta}$ generates for $n=1$

$$
h_{t}+h_{\theta \theta t}=\frac{1}{4}\left[\left(h+h_{\theta \theta}\right)\left(h^{2}+h_{\theta}^{2}\right)\right]_{\theta} .
$$

This is the dual counterpart of the $\mathrm{mKdV}$ equation considered by Olver and Rosenau (equation (25) in [14]). In this same work we recognize (up to multiplicative constants) equation (16) as the Lagrange transform of the mKdV equation (equation (28) in [14]). From (16) (or (18)) if we make the identification $\kappa=1 / \rho$ we obtain

$$
\rho_{t}=\frac{1}{2}\left(\rho^{-2}\right)_{\theta \theta \theta}+\frac{1}{2}\left(\rho^{-2}\right)_{\theta},
$$

called Casimir equation for the modified compacton hierarchy (up to multiplicative constants it is the equation (27) in [14]). It is implicit in [14] (due to the role played by the $\mathrm{mKdV}$ equation) that (34) and the CQ equation (15) are related by the Miura transformation $\rho=u+u_{x x}$. Using this transformation the bi-Hamiltonian structure of (34) follows from the bi-Hamiltonian structure of the CQ equation derived in Section 3 and they yield

$$
\begin{array}{ll}
\mathcal{D}_{1}=\partial+\partial^{3}, & H_{2}=-\frac{1}{2} \int d \theta \rho^{-1}, \\
\mathcal{D}_{2}=\partial \rho \partial^{-1} \rho \partial, & H_{1}=-\frac{1}{8} \int d \theta\left(\rho^{-3}-4 \rho^{-5} \rho_{\theta}^{2}\right) .
\end{array}
$$

The input of this bi-Hamiltonian structure was used in [13] for the derivation of the bi-Hamiltonian structure of the CQ equation. In fact they transformed $\mathcal{D}_{1}, H_{2}$ back to the $\mathrm{CQ}$ equation variables and the second Hamiltonian structure was obtained by a factorization of the recursion operator (6).

\section{Conclusion:}

In this paper, the transformations used by Sakovich to map the CQ and mKdV equations into each other were shown to have a nice geometrical interpretation. Namely, they follow from a isoperimetric curve motion in the Euclidean plane. Also, the bi-Hamiltonian structure of the system was obtained without any knowledge of its recursion operator or other known result of equations associated with the system under consideration. Finally, we have derived a hierarchy of equations, symmetries and equations associated with the CQ equation system which have appeared in the literature previously. 


\section{Acknowledgments}

We would like to thank the authors of [13] for sending us a copy of their paper. This work was supported by $\mathrm{CNPq}$ (Brazil).

\section{References}

[1] H. Hasimoto, J. Fluid Mech. 51, 477 (1972).

[2] G.L Lamb, J. Math. Phys. 18, 1654 (1977).

[3] M. Lakshmanan, J. Math. Phys. 20, 1667 (1979).

[4] J. Langer and R. Perline, J. Nonlinear Sci. 1, 71 (1991).

[5] A. Doliwa and P. M. Santini, Phys. Lett. A 185, 373 (1994).

[6] K. Nakayama, J. Phys. Soc. Jpn. 67, 3031 (1998); K. Nakayama, J. Phys. Soc. Jpn. 68, 3214 (1999).

[7] M. Gürses, Phys. Lett. A 241, 329 (1998).

[8] R. E. Goldstein and D. M. Petrich, Phys. Rev. Lett. 67, 3203 (1991).

[9] K. Nakayama, H. Segur and M. Wadati, Phys. Rev. Lett. 69, 2603 (1992).

[10] K.-S. Chou and C. Qu, Physica D 162, 9 (2002).

[11] K.-S. Chou and C. Qu, J. Nonlinear Sci. 13, 487 (2003).

[12] S. Yu. Sakovich, Phys. Lett. A 314, 232 (2003).

[13] W. X. Ren and Alatancang, Commun. Theor. Phys. 48, 211 (2007).

[14] P. J. Olver and P. Rosenau, Phys. Rev. E 53, 1900 (1996).

[15] H. W. Guggenheimer, Differential Geometry (Dover Publications, New York, 1973).

[16] J. C. Brunelli and G. A. T. F. da Costa, J. Math. Phys. 43, 6116 (2002); J. C. Brunelli, Phys. Lett. A 353, 475 (2006); J. C. Brunelli, J. Math. Phys. 46, 123507 (2005).

[17] P. A. M. Dirac, Lectures on Quantum Mechanics, Belfer Graduate School of Science Monographs, vol. 2 (New York, 1964); K. Sundermeyer, Constrained Dynamics, Lecture Notes in Physics, vol. 169 (Springer, Berlin, 1982).

[18] P. J. Olver, Applications of Lie Groups to Differential Equations, 2nd ed. (Springer, Berlin, 1993). 
[19] F. Magri, J. Math. Phys. 19, 1156 (1978).

[20] M. Adler, Invent. Math. 50, 219 (1979).

[21] J. C. Brunelli, Comput. Phys. Commun. 163, 22 (2004).

[22] A. F. Cheviakov, Comput. Phys. Commun. 176, 48 (2007). 